\title{
COVID-19 E DOENÇAS CRÔNICAS: IMPACTOS E DESDOBRAMENTOS FRENTE À PANDEMIA
}

\author{
COVID-19 AND CHRONIC DISEASES: IMPACTS \\ AND DEVELOPMENTS BEFORE THE PANDEMIC
}

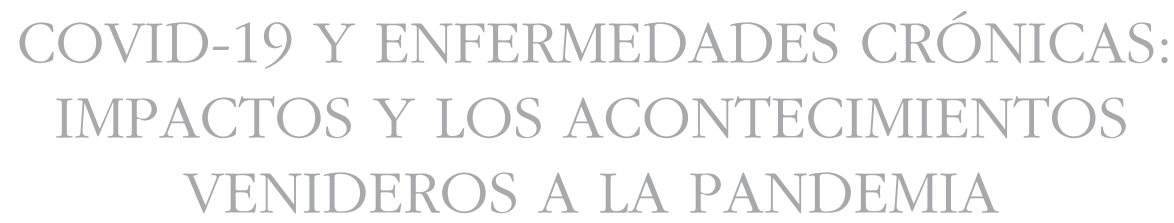

Fernanda Matheus Estrela ${ }^{1}$

Moniky Araújo da Cruz ${ }^{2}$

Nadirlene Pereira Gomes ${ }^{3}$

Milena Arão da Silva Oliveira ${ }^{4}$

Rebeca dos Santos Santos

Júlia Renata Fernandes Magalhães ${ }^{6}$

Lilian Conceição Guimarães de Almeida $^{7}$

Como citar este artigo: Estrela FM, Cruz MA, Gomes NP, Oliveira MAS, Santos RS, Magalhães JRF, et al. Covid-19 e doenças crônicas: impactos e desdobramentos frente à pandemia. Rev baiana enferm. 2020;34:e36559.

Objetivo: refletir sobre o elevado risco de complicações da COVID-19 em pessoas portadoras de doenças crônicas não transmissíveis, bem como os possíveis desdobramentos para aqueles acometidos pela doença. Método: revisão narrativa, desenvolvida com base em artigos publicados em periódicos e documentos de órgãos oficiais nacionais. Resultados: o estudo permitiu a reflexão acerca da vulnerabilidade das pessoas com doenças crônicas no contexto de pandemia pelo novo coronavírus, bem como os impactos para esse público. Esse cenário impacta substancialmente nos serviços de saúde, e sinaliza para a necessidade de uma atenção diferenciada no que tange as medidas de prevenção. Conclusão: o atual cenário apresenta-se com muitos desafios para a população, o que requer ações de autocuidado e atendimento às medidas de prevenção que visem o resguardo individual e coletivo.

Descritores: Covid-19. Pandemia. Doença Crônica.

Objective: to reflect on the high risk of complications of COVID-19 in people with chronic non-communicable diseases, as well as the possible consequences for those affected by the disease. Method: narrative review, developed based on articles published in journals and documents of official national agencies. Results: the study allowed the reflection about the vulnerability of people with chronic diseases in the context of the new coronavirus pandemic, as well as the impacts to this population. This scenario substantially impacts health services, and highlights the need for a differentiated attention regarding the prevention measures. Conclusion: the current scenario presents itself with many challenges for the population, which requires self-care actions and meetings preventive measures aimed at the individual and collective safety.

Descriptors: Covid-19. Pandemic. Chronic Disease.

\footnotetext{
Enfermeira. Doutora em Enfermagem. Professora da Universidade Estadual de Feira de Santana. Feira de Santana, Bahia, Brasil. nanmatheus@yahoo.com.br. http:// orcid.org/0000-000 I-750 I-6187.

Enfermeira. Universidade Federal da Bahia. Salvador, Bahia, Brasil. http://orcid.org/0000-0003-2955-5408.

Enfermeira. Doutora em Enfermagem. Professora Associada da Universidade Federal da Bahia. Salvador, Bahia, Brasil. http://orcid.org/0000-0002-6043-3997.

Enfermeira. Universidade Federal da Bahia. Salvador, Bahia, Brasil. http://orcid.org/0000-0003-3933-99|X.

Estudante de Enfermagem. Universidade Federal da Bahia. Salvador, Bahia, Brasil. http://orcid.org/0000-0002-2584- I I 78.

Enfermeira. Mestre em Enfermagem. Universidade Federal da Bahia. Salvador, Bahia, Brasil. http://orcid.org/0000-0003-0631-2374.

Enfermeira. Doutora em Enfermagem. Professora Adjunta da Universidade Federal da Bahia. Salvador, Bahia, Brasil. http://orcid.org/0000-000 I-6940-9/87.
} 
Objetivo: reflexionar sobre el alto riesgo de complicaciones de la COVID-19 en personas con enfermedades crónicas no transmisibles, asi como las posibles consecuencias para los afectados por la enfermedad. Método: revisión narrativa, desarrollada sobre la base de artículos publicados en revistas y documentos oficiales de organismos nacionales. Resultados: el estudio permitió la reflexión acerca de la vulnerabilidad de las personas con enfermedades crónicas en el contexto de la pandemia del nuevo coronavirus, asi como los impactos de esta población. Esta situación repercute considerablemente en los servicios de salud, y señala la necesidad de una atención diferenciada con respecto a las medidas de prevención. Conclusión: el escenario actual se presenta con muchos desafios para la población, que requiere acciones de autocuidado y el atendimiento a las medidas preventivas encaminadas a la protección individual y colectiva.

Descriptores: Covid-19. Pandemia. Enfermedad Crónica.

\section{Introdução}

Desde o surgimento da COVID-19, os números de casos crescem exponencialmente com impactos para a sociedade, a economia e a saúde dos indivíduos. Em sua forma mais grave atinge alguns grupos vulneráveis, como os portadores de doença crônica não transmissível (DCNT), o que sinaliza a necessidade de discussão acerca desse grupo, de modo a compreender e superar os inúmeros desafios que permeiam esse cenário.

Contextualizando, a COVID-19 é uma doença infecciosa causada pelo SARS-CoV-2, um novo coronavírus identificado na China, em dezembro de 2019. Diante do cenário de pandemia, anunciado em março de 2020, foram realizados alguns estudos, tendo sido identificado que pessoas com fragilidades no sistema imunológico compunham os grupos de riscos. Nestes, insere-se portadores de DCNT, como pacientes com diagnóstico de cardiopatias, hipertensão, diabetes, doença pulmonar obstrutiva crônica (DPOC), doenças renais e neurológicas ${ }^{(1-3)}$. Tais grupos de risco relacionam-se com índices de agravamento presentes em sua maioria nessa população.

Ser portador de DCNT tem estado relacionado às elevadas taxas de morbimortalidade quando associada à infecção por SARS-CoV-2. No Brasil, das pessoas que evoluíram a óbito por COVID-19, 70\% eram portadoras de DCNT; entre essas e as que tiveram recuperação, a maioria necessitou de leitos em Unidade de Terapia Intensiva (UTI) ${ }^{(4)}$. Vale lembrar que, para além da associação entre a prevalência de casos graves de COVID-19 e a presença de DCNT, novas complicações podem surgir em indivíduos sem histórico dessas doenças, visto que ainda não há informações completas sobre a história natural da infecção pelo SARS-CoV-2, nem manejos clínicos ideais para atender os pacientes em todas suas especificidades clínicas.

Uma das ações realizadas para conter a disseminação da doença versa sobre o isolamento social, que vem dividindo opiniões pelos altos impactos que essa medida ocasiona na sociedade, privando as pessoas do ir e vir e, sobretudo, na economia. Contudo, as taxas de incidência da COVID-19 estão se mantendo em níveis relativamente mais baixos do que o esperado, justamente devido à aplicação dessas medidas. Estudos apontam que se o isolamento social não fosse adotado, o índice de contaminação da população mundial poderia chegar a um intervalo de $60 \%$ a $80 \%$, como afirmado por um dos principais epidemiologistas de Hong Kong, Dr. Gabriel Leung, em uma reunião com a Organização Mundial da Saúde ${ }^{(5)}$. Diante disso, a não obediência a essas estratégias põe em risco não só a vida do indivíduo como também de toda a comunidade ao seu redor, o que afeta pessoas saudáveis, com maiores impactos naquelas que possuem doenças preexistentes, situação que, por sua vez, sobrecarrega os serviços de saúde.

Diante disso, faz-se necessária uma abordagem sobre os cuidados à população de modo a evitar a disseminação e a transmissão do SARS-CoV-2, sobretudo em portadores de patologias crônicas, tendo em vista todos os desafios e as consequências resultantes desse processo. 
Dessa forma, o objetivo deste ensaio é refletir sobre o elevado risco de complicações da COVID-19 em pessoas portadoras de doenças crônicas não transmissíveis e seus desdobramentos na população em geral, com ênfase nos cuidados.

\section{Método}

Trata-se de revisão narrativa, desenvolvida com base em artigos publicados em periódicos e documentos de órgãos oficiais. Este método permite descrever o estado da arte, de modo a sintetizar o conhecimento já exposto na literatura, somado às reflexões propostas pelos autores. Tal metodologia contribui para a discussão sobre um tema e é indicada para temáticas que necessitam de maiores aprofundamentos, assim como a COVID-19.

\section{Resultados e Discussão}

O estudo permitiu a reflexão acerca da vulnerabilidade das pessoas com doenças crônicas no contexto de pandemia pelo novo coronavírus, bem como os impactos para esse público. Esse cenário impacta substancialmente nos serviços de saúde, o que sinaliza para a necessidade de uma atenção diferenciada no que tange às medidas de prevenção.

\section{A vulnerabilidade das doenças crônicas diante da infecção pelo novo coronavírus}

As DCNT abrangem, além do câncer, outras enfermidades que afetam os sistemas respiratório, cardiovascular, endócrino e renal, configurando-se em uma das principais causas de morbimortalidade no mundo. Analisando sob a ótica dos índices de transmissão e agravamento da infecção pelo SARS-CoV-2, estudos epidemiológicos recentes revelaram que as pessoas portadoras de hipertensão, diabetes, doença pulmonar obstrutiva crônica e doenças renais têm maior probabilidade de desenvolver as formas mais graves da COVID-19, resultando em maiores taxas de morbimortalidade nessa população ${ }^{(6)}$.
Faz-se necessário a compreensão da fisiologia, dos determinantes sociais e econômicos, para que sejam pensadas estratégias de prevenção e enfrentamento para esse grupo populacional.

No que tange ao mecanismo fisiopatológico, associado ao agravamento da infecção pelo SARS-CoV-2, ressalta-se que apesar das evidências não serem de alto nível, aponta-se a participação da enzima conversora de angiotensina-2 (ECA2) nas células endoteliais. Esta é presente no coração, nos rins e nos pulmões, sendo a principal responsável pela entrada do novo coronavírus nas células da pessoa infectada, mediante a ativação de glicoproteínas e a clivagem da ECA2 por proteases ${ }^{(7)}$. Sendo assim, a regulação positiva dessa enzima ocorre em pacientes com doenças cardiovasculares, diabetes e hipertensão que fazem tratamento com inibidores da ECA2 e bloqueadores dos receptores da angiotensina II do tipo I, contribuindo para o crescimento exponencial de células infectadas e a rápida evolução da COVID-19 nessa população ${ }^{(7)}$. Além disso, indivíduos que convivem com DPOC e asma mal controlada também possuem possibilidade aumentada de agravamento da infecção pelo novo coronavírus, visto que esse grupo já apresenta fragilidades no sistema respiratório, que consiste no principal alvo de ataque do SARS-CoV-2 nas formas mais graves da doença.

Para além de fatores biológicos, ressalta-se que o elevado índice de DCNT no Brasil está amplamente relacionado aos estilos de vida adotados pela população, havendo interferência de determinantes sociais e fatores econômicos. No Brasil, assim como na maioria dos países em desenvolvimento, a elevada taxa de prevalência das DCNT revela que esses agravos atingem indiscriminadamente pessoas pertencentes a qualquer classe social, gênero, raça/cor e idade, afetando de forma mais intensa grupos vulneráveis ligados à baixa escolaridade, pobreza e raça negra. Essa realidade evidencia que, em um país marcado pela desigualdade social, não são apenas as características fisiopatológicas que influenciarão no agravamento da infecção pelo novo coronavírus, mas, também, os marcadores 
sociais. Essas diferenças poderão atuar como fator de risco para o processo, sendo de fundamental importância a elaboração de medidas políticas voltadas para esse grupo de maior vulnerabilidade nos âmbitos sociais, econômicos e de saúde.

Dessa forma, é necessário que sejam pensadas ações de educação em saúde, voltadas para hábitos de vida e alimentação equilibrada e saudável, prática de atividade física e uso de medicações de uso contínuo para o controle das doenças crônicas. Entretanto, essa não é a realidade brasileira devido ao baixo grau de escolaridade, aos trabalhos em ambientes insalubres com remuneração insuficiente, e dificuldade para se alimentar, no mínimo, com 3 refeições diárias. Todo esse cenário de desigualdade socioeconômico ainda é agravado pela dificuldade de acesso aos serviços de saúde, decorrente da baixa cobertura da atenção básica e das falhas na organização da oferta da assistência.

Diante disso, as demandas de saúde dessa população não conseguem ser atendidas de maneira integral, justa e universal indo contra os princípios estabelecidos pelo Sistema Único de Saúde (SUS). Com as necessidades de saúde negligenciadas, a qualidade de vida dos grupos menos favorecidos tende a não ser das melhores, levando a maior prevalência de doenças crônicas que fragilizam o organismo e enfraquecem o sistema imunológico. Sendo assim, em um contexto de pandemia pela COVID-19, garantir o acesso aos serviços de saúde e à informação, fornecer condições básicas de educação, moradia, emprego e alimentação resultam não apenas na melhoria da qualidade de vida desse grupo, mas também na diminuição do processo de contaminação dos indivíduos e na menor sobrecarga do sistema de saúde.

\section{Impactos físicos, psicológicos e sociais da COVID-19 nos portadores de doenças crônicas}

Considerando os portadores de DCNT enquanto grupo de risco para a COVID-19, como indicado pelo Ministério da Saúde (MS), cabe refletir sobre as repercussões de ordem física, psicológica e social que esse grupo está exposto quando infectado pela doença.

O cenário, por si só, acarreta uma mistura de sentimentos a toda a população, mas, em se tratando de doentes crônicos, há um aumento pelas incertezas e o potencial de agravamento. Alguns sintomas como depressão, ansiedade, distúrbios no sono, entre outros podem ser percebidos. Além disso, esses sintomas psicológicos podem ser somatizados levando à cefaléia, epigastralgia, taquicardia, falta ou excesso de apetite e outras manifestações clínicas que tornam, não só passar pela pandemia, mas também, o processo de recolhimento ainda mais doloroso. O surgimento desses sintomas psicológicos e físicos forma um cenário favorável para a instabilidade dos mecanismos fisiológicos do organismo, trazendo consequências, como o aumento da pressão arterial, a elevação dos níveis glicêmicos e a maior ocorrência de crises asmáticas, o que agrava o quadro daqueles que já possuem doenças crônicas.

O medo de serem infectados pelo SARS-CoV-2 gera desafios em alguns portadores de doenças crônicas, a exemplo da necessidade de se deslocarem de seus domicílios. O fato de ir ao trabalho, comprar suprimentos e, principalmente, adquirir as medicações de uso contínuo leva esses indivíduos a temerem pela sua própria vida e pela saúde de seus contatos mais próximos. Nesse contexto de pandemia, as consultas eletivas tiveram que ser suspensas, sendo atendidos apenas os casos de urgência e emergência. Como esse acompanhamento não é de emergência, muitos portadores têm suas consultas e exames cancelados, o que interfere diretamente na vigilância e no controle da doença, vulnerabilizando ainda mais essa população. Dessa forma, urge que sejam reorganizados os serviços de saúde frente a situações de calamidade pública, como a que estamos vivendo, fornecendo opções de acompanhamento e cuidado em saúde, adequados à realidade de cada pessoa.

Considerando os impactos diversos para os portadores de doenças crônicas, o governo 
brasileiro traçou algumas ações de modo a minimizar a propagação da doença, a exemplo da prorrogação da validade das receitas de medicamentos de uso contínuo para seis meses, diminuindo as transitações com essa finalidade. A orientação do uso de máscaras para a população em geral contribuiu para a proteção dos grupos vulneráveis, além da orientação do afastamento de pessoas com comorbidades das suas atividades laborais presenciais e a possibilidade de denúncia das instituições que fornecem condições básicas de trabalho nesse contexto. Em que pese as medidas já adotadas, faz-se necessário que sejam discutidos os desafios dos serviços de saúde nos diferentes níveis de atenção para com esse público.

\section{Desafios para os serviços de saúde em seus diferentes níveis de atenção}

A elevada prevalência das DCNT no cenário brasileiro revela a necessidade de medidas específicas com foco nesse nicho populacional para proteção dos indivíduos e evitar o colapso da rede assistencial. A esse respeito, o Ministério da Saúde publicou protocolos de manejo clínico da COVID-19 com orientações para a assistência e o monitoramento desse grupo de risco. A Atenção Primária à Saúde (APS) foi inserida nesse contexto com alguns objetivos, tal qual atender os casos considerados leves e estabilizar e encaminhar os casos mais graves da COVID-19. Contudo, sua potência de ação pode ser exercida mediante o acompanhamento dos pacientes portadores de doenças crônicas residentes em suas áreas adscritas por meio do teleatendimento ou das visitas domiciliares em situações excepcionais. Dessa forma, diversas estratégias precisam ser desenvolvidas para o maior alcance da vigilância em saúde desses pacientes com risco, configurando-se então como foco de atenção dos gestores da APS.

A articulação necessária para colocar em prática essas estratégias será coordenada principalmente pelas enfermeiras devido ao seu potencial administrativo e gerencial, que as colocam no papel de liderança e na condução das atividades dos agentes comunitários de saúde (ACS). Tendo em vista a extensão da unidade de saúde na comunidade como essência do trabalho do ACS, torna-se prioritário adotar ações de monitoramento dos pacientes com doenças crônicas em relação ao aparecimento de sinais e sintomas da COVID-19, visando a rápida intervenção da equipe de saúde e a consequente diminuição das complicações advindas do atraso assistencial a esses pacientes. Infelizmente, toda essa potência não está disponível para ser utilizada, considerando haver limitações, como a baixa cobertura da Estratégia Saúde da Família (ESF) e o desfalque das equipes, além da parcela populacional que desconhece o diagnóstico e, portanto, não realiza o tratamento, estando ainda mais vulneráveis às complicações por COVID-19.

Frente a esse contexto de aumento da demanda, para não sobrecarregar ainda mais o sistema de saúde e visando priorizar o atendimento, é importante a realização do Acolhimento com Classificação de Risco (ACCR). A atenção deve voltar-se aos sintomáticos respiratórios nos três níveis de atenção, contudo ampliando ainda mais o olhar no que diz respeito aos fatores de risco que contribuem com as complicações da COVID-19.

Desse modo, é possível agilizar o atendimento dentro da própria unidade ou o encaminhamento para um serviço de maior complexidade. Especificamente dentro da atenção secundária, é preciso ser repensado um fluxo de atendimento diferenciado nas unidades de pronto-atendimento, para evitar o cruzamento de pacientes sintomáticos respiratórios com os demais. Essa organização é de extrema importância para a proteção dos pacientes com DCNT que necessitem de atendimento nessas unidades de saúde, seja por descompensação dos sinais clínicos ou por outro motivo qualquer.

A necessidade de atendimento de forma específica nesta pandemia pode nos trazer de legado a possibilidade de novos desenhos estruturais para as unidades de saúde que facilitem o fluxo de acesso e o tratamento de pacientes potencialmente infectados, sem renunciar à conquista 
da porta única nos estabelecimentos de saúde, advinda com o SUS.

Essas ações, bem como a implementação de orientações outras preconizadas pelo Ministério da Saúde, podem ser dificultadas pela escassez de equipamentos de proteção individual (EPI) e deficiências na estrutura física. Dessa forma, a equipe de gestão da unidade de saúde necessita de empenho máximo, de acordo com as possibilidades, para não permitir que a unidade de saúde se torne foco de contaminação. Urge que sejam traçados novos exemplos criativos e resolutivos de experiências exitosas tanto na atenção aos pacientes com coronavírus quanto na proteção dos demais pacientes, com atenção aos mais vulneráveis, em unidades que realizam atendimento não exclusivo para a COVID-19.

Não diferente dos demais níveis, a atenção especializada também enfrenta desafios para a assistência aos pacientes de coronavírus com comorbidades. É preciso uma equipe multiprofissional para o atendimento integral e individualizado aos portadores de doenças crônicas para avaliação da manutenção da terapêutica já utilizada pelo paciente e as possíveis interações medicamentosas. Além da associação com comorbidade, que vulnerabilizam os pacientes à infecção pela COVID-19, existe a possibilidade da agudização das DCNT num movimento cíclico, levando a complicações e dificuldade no manejo desses pacientes.

Entende-se que novas pesquisas devem ser desenvolvidas para o manejo clínico realizado nesses pacientes de forma a apresentarem efetividade e contribuições para a estabilização clínica de forma rápida e segura. Para que sejam minimizadas as complicações para esse grupo populacional, o MS orienta o monitoramento contínuo e medidas de suporte necessárias à situação apresentada.

Nesse sentido, a enfermagem mostra-se essencial no cuidado aos pacientes por acompanhá-los de perto e realizar o controle dos sinais vitais de forma sistemática em sua rotina de trabalho. Entender a identificação precoce dos sinais e sintomas apresentados pelo paciente para um desfecho favorável é de suma importância a comunicação clara e efetiva entre a equipe multidisciplinar.

O estudo limita-se pelo próprio contexto, que frente aos estudos incipientes não permite avanços nas discussões acerca de todos os impactos que podem ser gerados para os indivíduos sadios ou portadores de comorbidades. Nesse quesito, são necessárias novas reflexões acerca da associação da COVID-19 com as doenças crônicas, ou ainda, com o desenvolvimento de outras doenças advindas após a recuperação por essa infecção.

\section{Conclusão}

Diante do cenário de pandemia e preocupações com o agravamento da COVID-19 aos portadores de DCNT, salientam-se as discussões sobre os impactos e possíveis desdobramentos. O atual cenário apresenta-se com muitos desafios para a população, o que requer ações de autocuidado e atendimento às medidas de prevenção que visem o resguardo individual e coletivo. A adoção de tais atos contribui para a redução da probabilidade da doença chegar aos grupos vulneráveis e, por sua vez, diminui os índices de morbimortalidade, enfrentamento que deve ser abraçado pela sociedade.

As medidas coletivas não só afetam a comunidade, mas impõem responsabilidades aos órgãos governamentais para que estruturem ações de prevenção e enfrentamento. Sendo assim, cabe que a gestão pública organize suas prioridades dando atenção aos públicos de risco, de modo que preserve a vida, mas também evite a sobrecarga dos serviços públicos. Sobretudo, este desafio abrange à área da saúde, que necessita estruturar sua rede para assistir à população e promover a saúde. Essas ações podem ser potencializadas com a capacitação dos profissionais, principalmente da enfermagem, que têm, em sua essência, as ações de educação em saúde.

Acredita-se que as reflexões propostas nesse trabalho possam cooperar para a ampliação do olhar aos doentes crônicos, enquanto grupo de risco, e assim melhorar as ações de cuidado voltadas a essa população. Além disso, espera-se 
que o entendimento acerca dessa correlação da nova doença com as comorbidades possa favorecer o desenho de orientações assertivas e eficientes para o manejo clínico dos pacientes portadores de doenças crônicas infectados pelo vírus, ou ainda, estratégias de prevenção para com a população geral.

\section{Colaborações:}

1 - concepção, projeto, análise e interpretação dos dados: Fernanda Matheus Estrela, Moniky Araújo da Cruz, Nadirlene Pereira Gomes, Milena Arão da Silva Oliveira, Rebeca dos Santos Santos, Júlia Renata Fernandes Magalhães e Lilian Conceição Guimarães de Almeida;

2 - redação do artigo e revisão crítica relevante do conteúdo intelectual: Fernanda Matheus Estrela, Moniky Araújo da Cruz, Nadirlene Pereira Gomes, Milena Arão da Silva Oliveira, Rebeca dos Santos Santos, Júlia Renata Fernandes Magalhães e Lilian Conceição Guimarães de Almeida;

3 - aprovação final da versão a ser publicada: Fernanda Matheus Estrela, Moniky Araújo da Cruz, Nadirlene Pereira Gomes, Milena Arão da Silva Oliveira, Rebeca dos Santos Santos, Júlia Renata Fernandes Magalhães e Lilian Conceição Guimarães de Almeida.

\section{Referências}

1. Brasil. Ministério da Saúde. Secretaria de Atenção Especializada à Saúde. Departamento de Atenção Hospitalar, Domiciliar e de Urgência. Diretrizes para diagnóstico e tratamento da Covid-19 [Internet]. Brasília (DF); 2020 [cited 2020 Apr 10]. Available from: https://portalarquivos.saude.gov.br/images/ pdf/2020/April/13/Diretrizes-COVID-13-4.pdf
2. Brasil. Ministério da Saúde. Secretaria de Atenção Primária à Saúde. Protocolo de manejo clínico do coronavírus (COVID-19) na atenção primária à saúde [Internet]. Brasília (DF); 2020 [cited 2020 Apr 10]. Available from: https:// www.saude.gov.br/images/pdf/2020/ marco/20/20200318-ProtocoloManejo-ver002.pdf

3. Wenzhong L, Hualan L. Covid-19: Attacks the 1-Beta Chain of Hemoglobin and Captures the Porphyrin to Inhibit Human Heme Metabolism. ChemRxiv. 2020;6 DOI: https://doi.org/10.26434/ chemrxiv.11938173.v6

4. Brasil. Ministério da Saúde. Centro de Operações de Emergência em Saúde Pública. Boletim COE COVID-19 n. 13 [Internet]. Brasília (DF); 2020 [cited 2020 Apr 23]. Available from: https:// portalarquivos.saude.gov.br/images/pdf/2020/ April/21/BE13---Boletim-do-COE.pdf

5. Coronavírus pode infetar mais de metade da população mundial se não for controlado, diz epidemiologista [Internet]. Expresso (Rio de Janeiro). 2020 fev 11 [cited 2020 Apr 29]; Available from: https://expresso.pt/internacional/ 2020-02-11-Coronavirus-pode-infetar-maisde-metade-da-populacao-mundial-se-nao-forcontrolado-diz-epidemiologista

6. Li X, Xu S, Yu M, Wang K, Tao Y, Zhou Y, et al. Risk factors for severity and mortality in adult COVID-19 inpatients in Wuhan. J Allergy Clin Immunol. 2020 Apr 12. DOI: https://doi. org/10.1016/j.jaci.2020.04.006

7. Pal R, Bhadada SK. COVID-19 and noncommunicable diseases. Postgrad Med J. 2020 Mar 30. DOI: http://dx.doi.org/10.1136/ postgradmedj-2020-137742

Recebido: 27 de abril de 2020

Aprovado: 11 de maio de 2020

Publicado: 8 de junho de 2020

A Revista Baiana de Enfermagem utiliza a Licença Creative Commons - Atribuição-NãoComercial 4.0 Internacional. https://creativecommons.org/licenses/by-nc/4.0/ Este artigo é de acesso aberto distribuído sob os termos da Licença Creative Commons (CC BY-NC).

Esta licença permite que outros remixem, adaptem e criem a partir do seu trabalho para fins não comerciais. Embora os novos trabalhos tenham de lhe atribuir o devido crédito e não possam ser usados para fins comerciais, os usuários não têm de licenciar esses trabalhos derivados sob os mesmos termos. 